Simulations of the Tilted Rocket Rig

LAWRENCE LIVERMORE NATIONAL LABORATORY
Experiments using the K-L RANS Model in the Ares Hydrodynamics Code

J. A. Greenough and M. Wickett 
This was work performed under the auspices of the U.S. Department of Energy by Lawrence Livermore National Laboratory under Contract DE-AC52-07NA27344.

This document was prepared as an account of work sponsored by an agency of the United States government. Neither the United States government nor Lawrence Livermore National Security, LLC, nor any of their employees makes any warranty, expressed or implied, or assumes any legal liability or responsibility for the accuracy, completeness, or usefulness of any information, apparatus, product, or process disclosed, or represents that its use would not infringe privately owned rights. Reference herein to any specific commercial product, process, or service by trade name, trademark, manufacturer, or otherwise does not necessarily constitute or imply its endorsement, recommendation, or favoring by the United States government or Lawrence Livermore National Security, LLC. The views and opinions of authors expressed herein do not necessarily state or reflect those of the United States government or Lawrence Livermore National Security, LLC, and shall not be used for advertising or product endorsement purposes. 


\title{
Simulations of the Tilted Rocket Rig Experiments using the K-L RANS Model in the Ares Hydrodynamics Code
}

\author{
J.A. Greenough and M. Wickett \\ LLNL, B-Division
}

August 27, 2012

\begin{abstract}
Numerical simulations of the tilted Rocket Rig Rayleigh Taylor Experiment, Case 110, are performed using the K-L RANS model in Ares hydrodynamics code. The effects of initialization, resolution, and the initial turbulent lengthscale are studied for K-L. The BHR model in the LANL code FLAG is compared to the K-L model in Ares for cases where we have the data. Some preliminary conclusions are made and a proposal for future work is given.

\section{Introduction}

As part of a joint LANL and LLNL RANS model comparison project, Case 110 from the Smeeton and Youngs AWE Report [1] was chosen as a test for the current generation of RANS models. The K-L model in the Ares code will be applied to this problem.

A formal Test Problem (TP) prescription is available at this writing as version 1. Some variances are made in this setup so that comparisons with the LANL FLAG code can be made easily. The TP document is being used for guidance on diagnostics for comparison and overall problem geometry and acceleration specification.
\end{abstract}

Figure 1 shows a schematic of the test problem. In this model it is the interface that is tilted rather than tilting the apparatus relative to the earth's gravitational field as was done in the experiment.

\section{Initial Conditions}

To facilitate comparisons with LANL, we adopt the following initial state and equation of state (EOS) prescription, without justification. The EOS for each fluid is taken to be an ideal gas with a gamma of 1.4. The initial conditions for Case110 are summarized in Table 1. 


\begin{tabular}{|l|l|l|l|l|}
\hline Case 110 & $\begin{array}{l}\text { Density } \\
(\mathrm{g} / \mathrm{cc})\end{array}$ & $\begin{array}{l}\text { Pressure } \\
(\mathrm{bar})\end{array}$ & $\begin{array}{l}\text { Temperature } \\
(\mathrm{K})\end{array}$ & gamma \\
\hline $\begin{array}{l}\text { Heavy fluid } \\
\text { (upper) }\end{array}$ & $1.89 \mathrm{e}-3$ & 0.1 & 300 & 1.4 \\
\hline $\begin{array}{l}\text { Light fluid } \\
\text { (lower) }\end{array}$ & $0.66 \mathrm{e}-3$ & 0.1 & 300 & 1.4 \\
\hline & $\mathrm{L}_{\mathrm{x}}=15 \mathrm{~cm}$ & $\mathrm{~L}_{\mathrm{y}}=24 \mathrm{~cm}$ & $\mathrm{~L}_{\mathrm{c}}=12 \mathrm{~cm}$ & \\
\hline
\end{tabular}

Table 1. Summary of initial conditions for Case 110 from reference [1].

The angle, $\theta$, shown in figure 1 is taken to be nominally 5 degrees. The acceleration applied by rocket motors is given in tabulated form in appendix A.

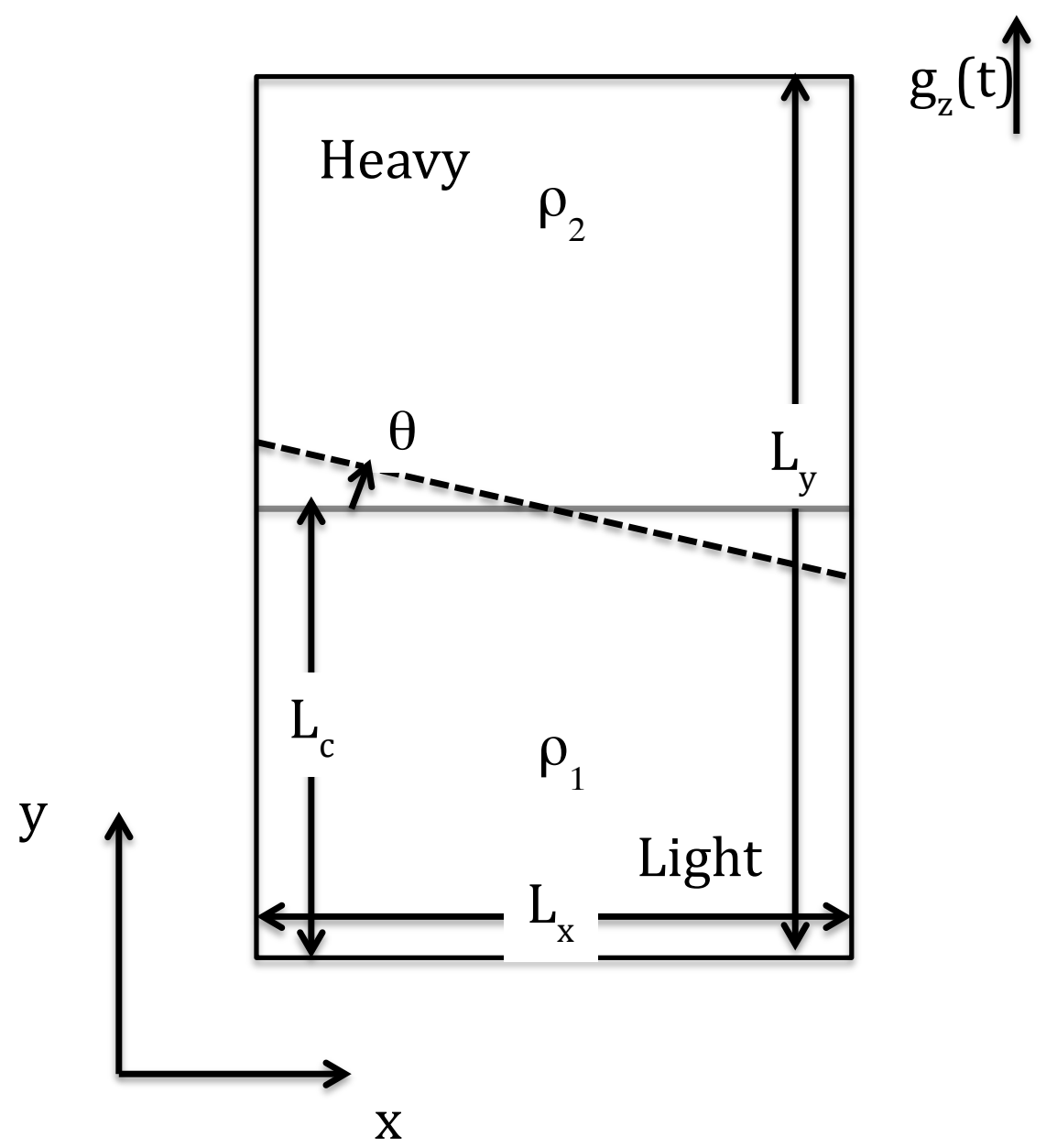

Figure 1. Schematic of the problem domain for the computational model of the tilted Rocket Rig test problem. See the text for the definitions of the parameters.

\section{The K-L RANS Model}

The K-L RANS model as implemented in the Ares code is given by the following set of equations as: 


$$
\begin{aligned}
& \rho \frac{d K}{d t}=-b_{t} L \sqrt{K} \frac{\nabla \rho \bullet \nabla p}{\rho}+c_{t} \mu_{t} \omega^{2}-p_{t} \nabla \bullet \vec{u}+v_{s} \tau_{i j} S_{i j}-d_{t} \frac{\rho K^{3 / 2}}{L}+\nabla \bullet\left(\frac{\mu_{t}}{k_{\text {num }}} \nabla K\right) \\
& \rho \frac{d L}{d t}=l_{1 t} \rho L \nabla \cdot \vec{u}+l_{2 t} \rho \sqrt{2 K} L \nabla \cdot \vec{u}+\nabla \cdot\left(\frac{\mu_{t}}{l_{\text {num }}} \nabla L\right) \\
& \rho \frac{d U}{d t}=b_{t} L \sqrt{K} \frac{\nabla \rho \bullet \nabla p}{\rho}-c_{t} \mu_{t} \omega^{2}-p \nabla \cdot \vec{u}+d_{t} \frac{\rho K^{3 / 2}}{L}+\nabla \bullet\left(\frac{\mu_{t}}{k_{\text {num }}} \nabla U\right) \\
& \rho \frac{d m_{r}}{d t}=\nabla \cdot\left(\frac{\mu_{t}}{S c} \nabla m_{r}\right) \\
& \rho \frac{d \vec{u}}{d t}=-\nabla\left(p+p_{t}\right)+v_{s} \nabla \bullet \tau_{i j} \\
& S_{i j}=\frac{1}{2}\left(\frac{\partial u_{i}}{\partial x_{j}}+\frac{\partial u_{j}}{\partial x_{i}}\right)-\frac{1}{3} \frac{\partial u_{k}}{\partial x_{k}} \delta_{i j} \\
& \tau_{i j}=2 \mu_{t} S_{i j}
\end{aligned}
$$

and also including the following definitions:

$$
\begin{gathered}
\mu_{t}=a_{t} \rho \sqrt{K} L \\
p_{t}=\frac{2}{3} \rho K
\end{gathered}
$$

for the turbulent viscosity and the turbulent pressure.

In order, these are equations for the unresolved turbulent kinetic energy $\mathrm{K}$ (equation 1), the turbulent lengthscale L (equation 2), the resolved specific internal energy $U$ (equation 3 ), the species mass fraction $m_{r}$ (equation 4 ) and the resolved velocity vector field (equation 5). Equations 6 and 7 give the strain-rate tensor and the viscous stress, respectively. There are a number of constants that must be specified in this model. We use the standard set of coefficients given in the Ares Users' Guide and summarized in Table 1.

\begin{tabular}{|c|c|c|}
\hline $\mathrm{a}_{\mathrm{t}}=1.414$ & $\operatorname{Pr}=1.0$ & $\mathrm{l}_{1 \mathrm{t}}=1.0$ \\
\hline $\mathrm{b}_{\mathrm{t}}=1.2$ & $\mathrm{Sc}=1.0$ & $\mathrm{l}_{2 \mathrm{t}}=1.0$ \\
\hline $\mathrm{c}_{\mathrm{t}}=0.0$ & $\mathrm{v}_{\mathrm{s}}=0.1$ & $\mathrm{k}_{\text {num }}=1.0$ \\
\hline $\mathrm{d}_{\mathrm{t}}=3.5$ & & $\mathrm{l}_{\text {num }}=0.5$ \\
\hline
\end{tabular}

Table 2. Summary of the standard set of coefficients and model constants for the KL RANS model in Ares. 
This set of coefficients is based on those obtained by Dimonte and Tipton [2] for their variant of the K-L model.

\section{Model Initialization}

There are several ways to initialize the K-L RANS model. In this study we show results using two variants of the simplest initialization method. Initial values for $\mathrm{K}$ and $\mathrm{L}$ are prescribed as constants over the entire field at $\mathrm{t}=0$. A desired feature of the model is that regardless of the finite values for $K$ and $L$ at $t=0$, there should be no instability growth if there is no applied acceleration. By construction of the model, if the initial value for $\mathrm{K}$ is too large and there is no applied acceleration, $\mathrm{K}$ will decay into the internal energy $\mathrm{U}$, to satisfy conservation of energy. Some diagnostics will not be sensitive to this effect, like the spike and bubble heights. But others like the integral of K over the domain are. For brevity, we will only show a few results with the initial $\mathrm{K}$ too large and focus on the other case were the initial value of $\mathrm{K}$ is "small enough" to not affect the results.

To control the model evolution, e.g. how much mixing there is, we vary the initial L. This is the typical use case where the initial $\mathrm{L}$ is varied until an acceptable match to data is obtained.

\section{Diagnostics}

The test problem description (version 1) [3] gives a set of diagnostics in section 3.1.1. In this study, we will provide the following quantities:

- Spike and bubble heights (NB: these are values that are obtained on the side walls of the computational domain). The cut-off values given in [3], 0.001, are used to calculate the heights.

- Integral mix width, W. We deviate from the official TP definition for this quantity so that it can be defined more naturally (i.e. we replace averaging with integration in the transverse directions and use integration only in the vertical applied acceleration direction).

- Integral of $\mathrm{K}$ and $\mathrm{U}$ over the domain.

- Mixedness, using our alternative definitions consistent with the integral mix width.

We define an averaging operator $<\mathrm{q}>$ as

$<q>(y, t)=\frac{1}{N_{x} N_{z}} \sum_{i, k} q(x, y, z, t)$

which represents averaging of $\mathrm{q}$ in the transverse (to the applied acceleration) directions. In figure 1, the z-direction is out of the page in the usual right-handed sense.

To construct $\mathrm{W}$, our definition of the integral mix width, we evaluate the following definite integral 


$$
W(t)=\int_{0}^{L_{y}}<f_{1}>(y, t)<f_{2}>(y, t) d y
$$

We note that this definition for $\mathrm{W}$ naturally has units of length and for finite amounts of initial mixing has a non-zero value.

To construct the Mixedness, we need to evaluate an addition integral, namely

$$
W_{2}(t)=\int_{0}^{L_{y}}<f_{1} f_{2}>(y, t) d y
$$

Then the Mixedness is given as

$$
\Theta(t)=\frac{W_{2}(t)}{W(t)}
$$

The integral of $\mathrm{K}$ and $\mathrm{U}$ are given in the usual form and have units of energy as

$$
\begin{aligned}
& T K E=\int_{0}^{L_{y}} \int_{0}^{L_{x}} \rho(x, y, t) K(x, y, t) d y d x \\
& I E=\int_{0}^{L_{y}} \int_{0}^{L_{x}} \rho(x, y, t) U(x, y, t) d y d x
\end{aligned}
$$

The spike and bubble heights are computed using Python scripts using the VisIt visualization and analysis package. Averaging operators are defined that implement the operator defined by equation (9). Then the curves are searched and interpolated to find the height at the cut-off value of 0.001 .

\section{Results}

Figures 2 and 3 show a set of Ares results, for bubbles and spikes, respectively, using the alternative initialization (small enough initial $\mathrm{K}$ ) and varying $\mathrm{L}$ compared to a result from the FLAG code running the BHR model. It should be pointed out that the Ares analysis has an artifact that is of order the mesh spacing that affects the maximal and minimal values. The maximal and minimal values should be the top and bottom wall values, but the current VisIt analysis gives values slight off the correct value by approximately half the vertical mesh spacing. We are working with the VisIt team to address this issue.

Overall the agreement for spike an bubble evolution is acceptable. The best agreement with FLAG is obtained for lower values of $L$.

A summary of the Integral Mix Width, $\mathrm{W}$, is shown in figure 4. Comparing the 101 and 201 resolution results, we appear to be near mesh convergence as there is only minor differences between the two cases. The FLAG result using the definitions in this report, denoted as "Alt. defn" in the figure legend, agree well with Ares result.

Figure 5 shows the results for this report's definition of Mixedness. Given an initial fixed initial condition, all of the Ares results give the same $t=0$ value. The degree of 
mixing is seen to decrease as the initial lengthscale $L$ decreases. For completeness, a result with the model off is shown for Ares and FLAG. The variations in these cases are probably due to numerical mixing during the remap phase of the calculation. As future work we should investigate this more closely.

Figure 6 shows the evolution of the integrated TKE. Using the alternative initialization in Ares for a set of initial L's, we obtain a family of curves that vary consistently. Increasing the initial L increases the integrated TKE and visa versa. The FLAG result, at intermediate and late times, appears to be growing at a faster rate than the Ares results. As future work, we will compare the model coefficients used in BHR to those in the Ares' K-L model and see it a choice there causes the difference.

Figure 7 shows the evolution of the integrated internal energy, IE. Increasing the Ares initial $L$ increases IE, so that overall we have a similar family of curves. The FLAG IE shows amplitude oscillations, that are larger than the ones from the Ares results, over the entire calculation and has a steeper slope at late time. We speculate that differences in the model coefficient are producing the difference.

\section{Conclusions and Future Work}

We have worked through the bulk of the major differences between our simulations and those at LANL with the FLAG code. Several iterations were necessary to finally be running the same problem as LANL. The EOS, intial state and diagnostic definitions had to be agreed to.

The agreement on lower order metrics like spike and bubble heights is good. The detailed diagnostics such as the TKE and IE evolution show a different power law evolution. We should pursue a comparison of the model coefficients to resolve that difference.

Additional recommended work items are:

- assessment of numerical mixing compared to modeled mixing

- further investigations on the early time integrated $\mathrm{K}$ decay differences. Possibly use the Barenblatt "burst" [4] analytic problem to explore that.

- Compare and contrast the model coefficient sets, which requires having the BHR coefficient set along with the as implemented equations. 


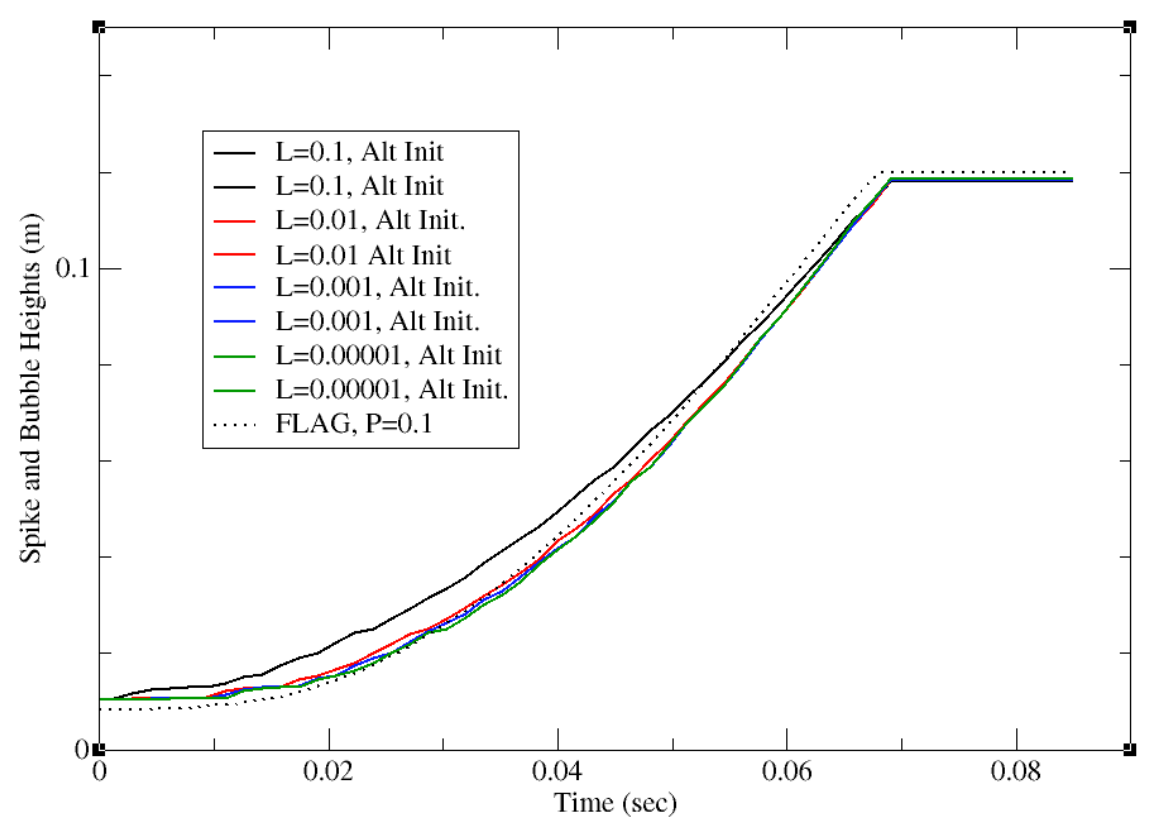

Figure 2. Bubble heights for Ares using the alternative initialization (small enough initial K) and varying the initial L compared to the LANL FLAG code result.

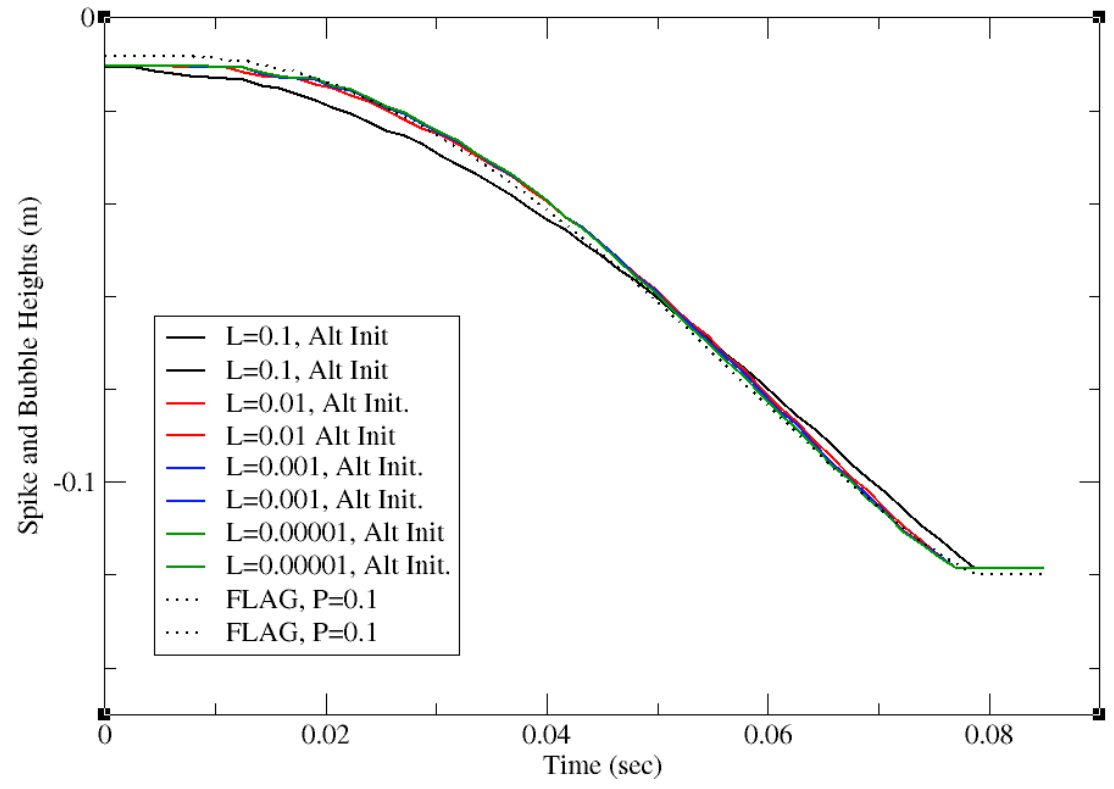

Figure 3. Spike heights for Ares using the alternative initialization (small enough initial $\mathrm{K}$ ) and varying the initial L compared to the LANL FLAG code result. 


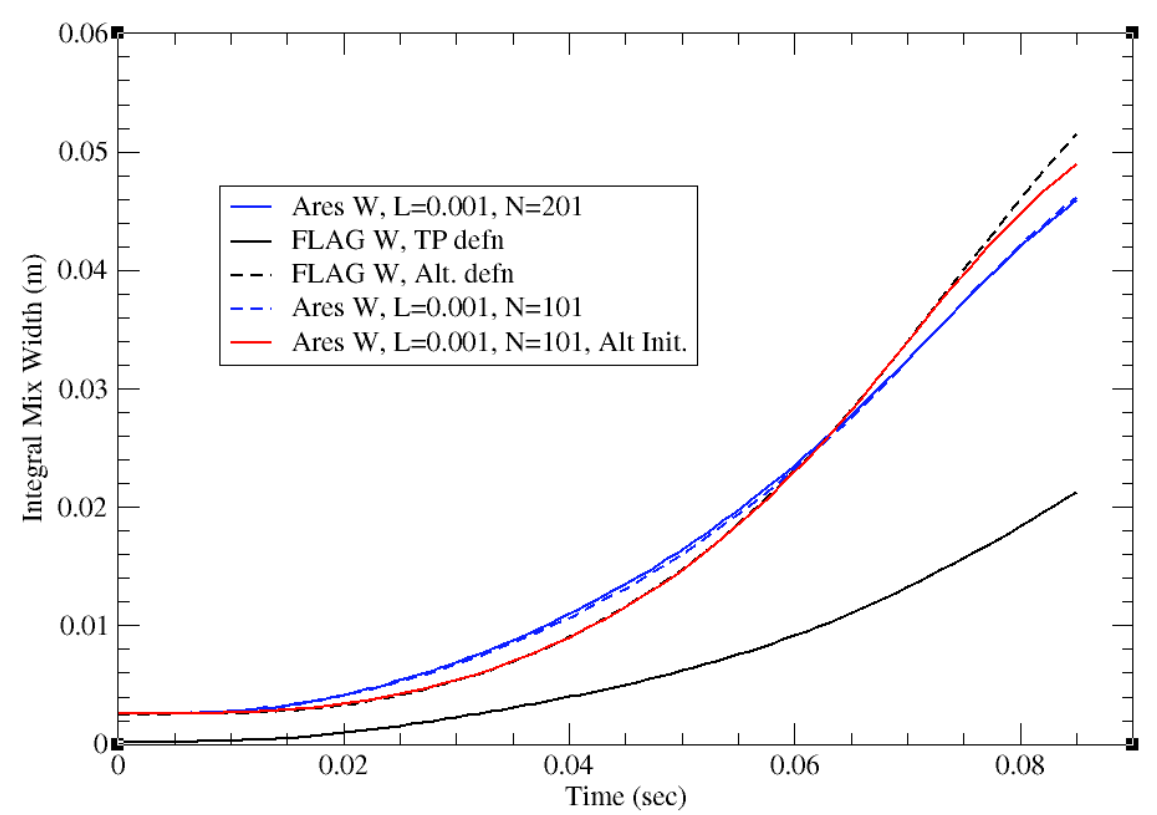

Figure 4. The Integral Mix Width for Ares using the alternative initialization (small enough initial $\mathrm{K}$ ) and base initialization (larger initial $\mathrm{K}$ so that $\mathrm{K}$ decays into $\mathrm{U}$ prior to the applied acceleration). Also shown is a resolution study using 101 and 201 vertical mesh points. For the FLAG results, the "Alt. defn" is directly comparable to the definitions in this report and what is computed from the Ares results.

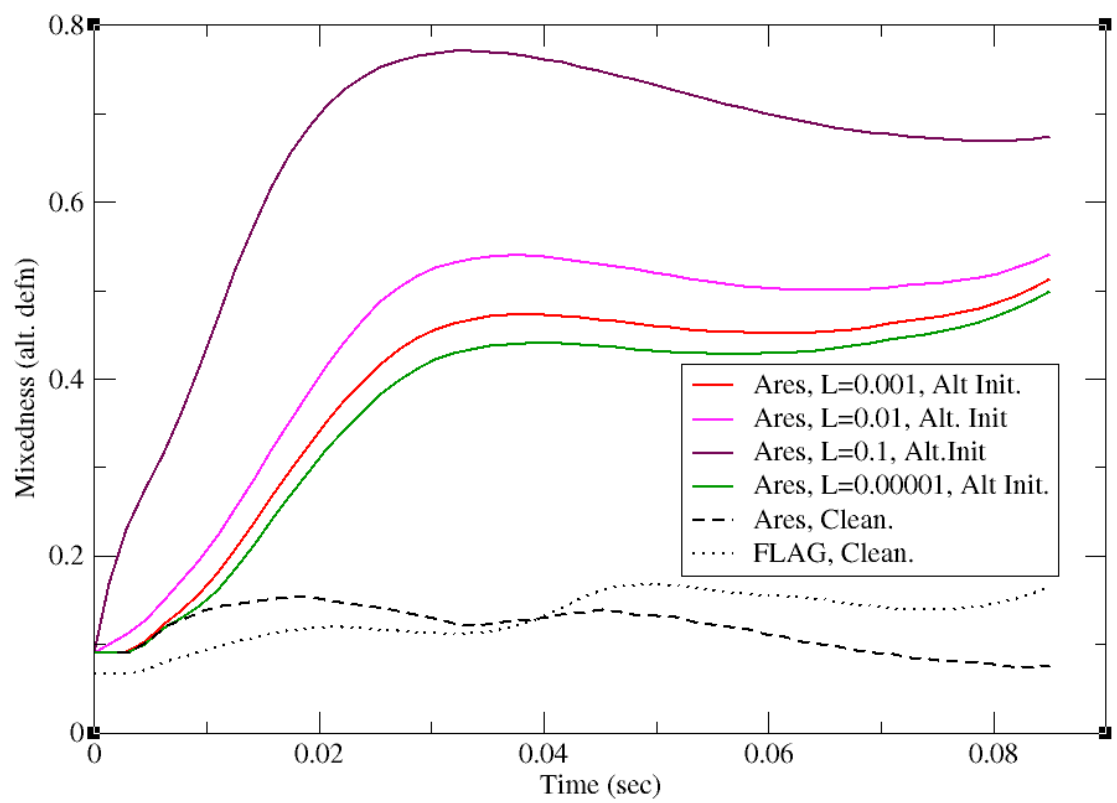

Figure 5. The Mixedness is shown for a set of initial L's, using the alternative initialization (small enough initial K), for Ares including a model off (denoted as "Clean" in the figure legend) from both codes. 


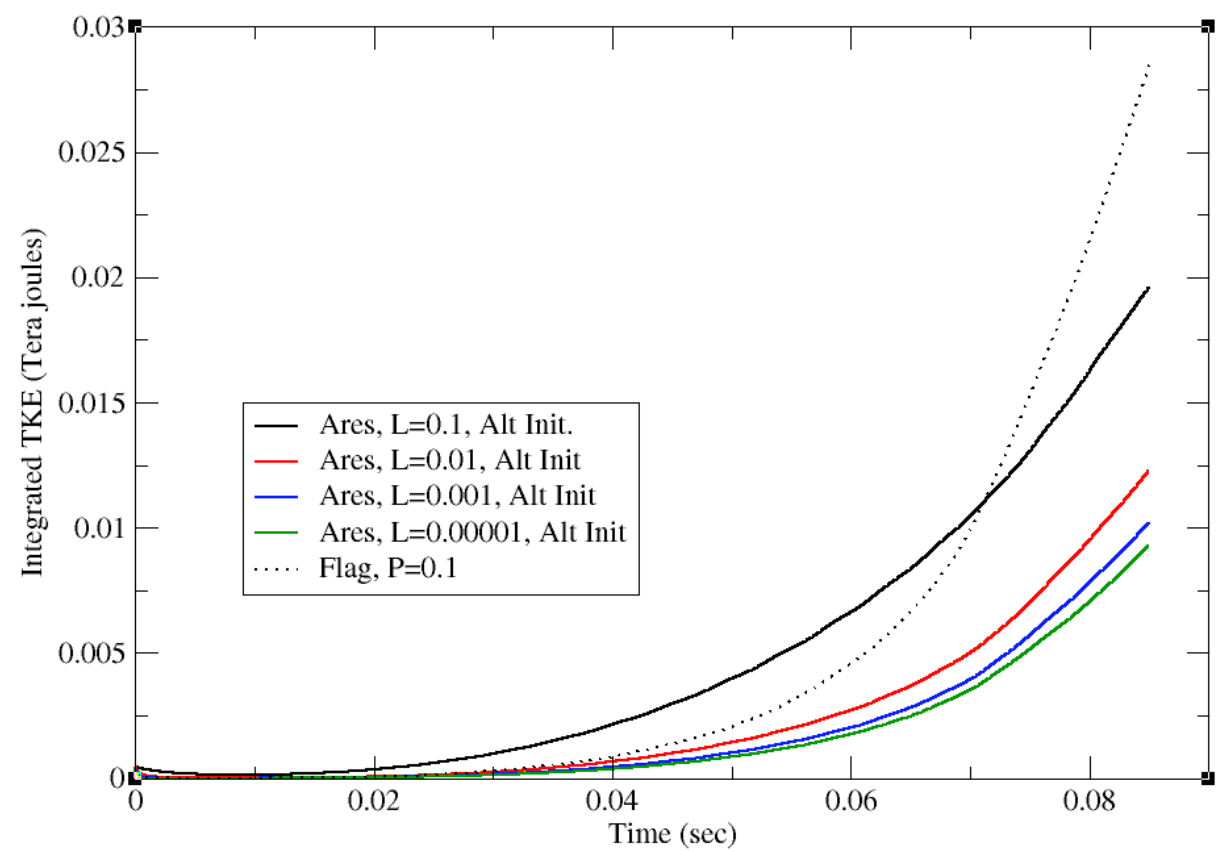

Figure 6. The integrated TKE is plotted using the alternative initialization in Ares for a set of different initial L's. For comparison we have a single FLAG result.

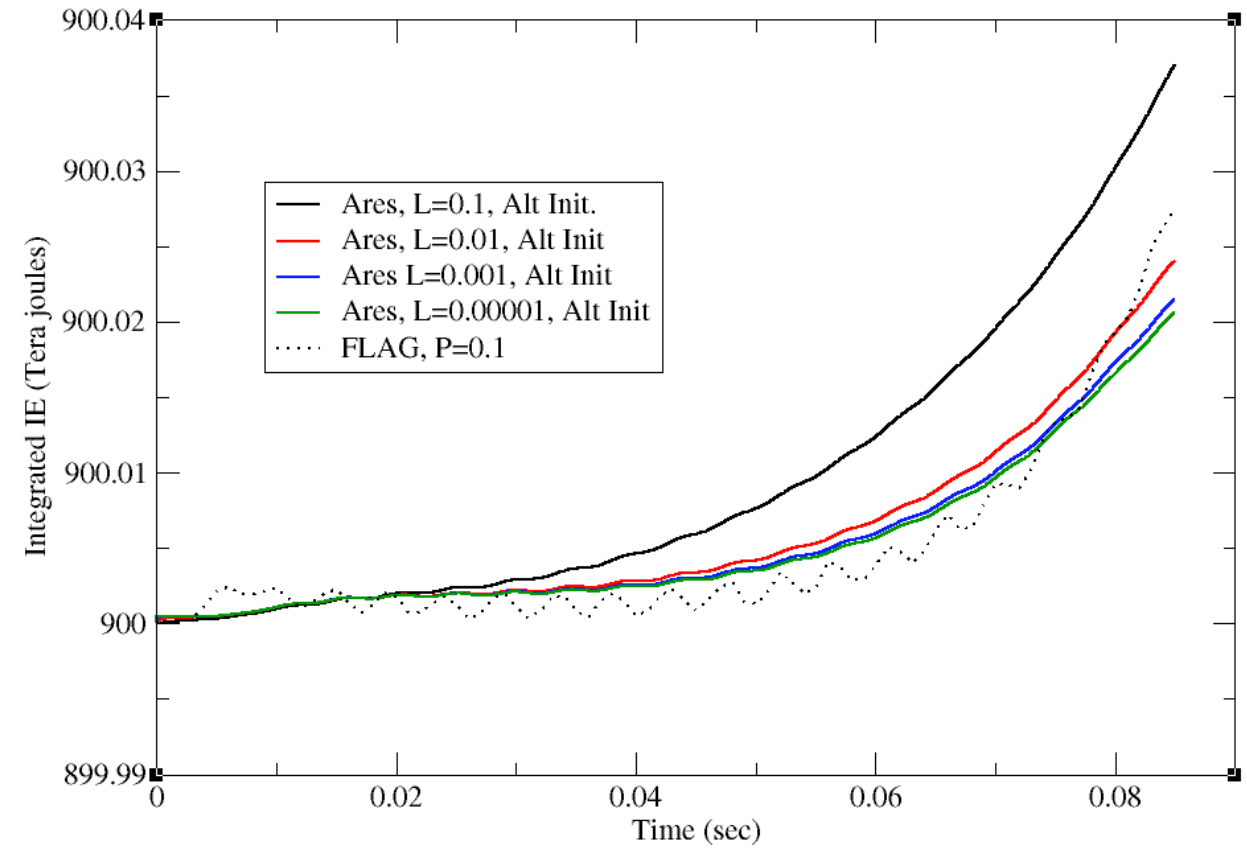

Figure 7. The integrated internal energy, IE, is plotted using the alternative initialization in Ares for a set of different initial L's. For comparison we have a single FLAG result. 


\section{References}

[1] V.S. Smeeton and D. L. Youngs, "Experimental investigation of the turbulent mixing by Rayleigh-Taylor instability, Part 3", AWE Report No. 0 35/87, 1987.

[2] G. Dimonte and R. Tipton, "K-L turbulence model for the self-similar growth of the Rayleigh-Taylor and Richtmeyer-Meshkov instabilities," Physics of Fluids, 18, 085101 (2006).

[3] M.J. Andrews, D.L. Youngs and D. Livescu, "Test problem: tilted Rayleigh-Taylor for 2-D mixing studies", LA-UR 12-24091, 2012.

[4] G.I. Barenblatt, "Self Similar Turbulence propagation from an instantaneous plane source", pp. 48-60. Nonlinear Dynamics and Turbulence, Interaction between Mathematics and Mechanics Series, Pitman. 
Appendix A. The time dependent acceleration for Smeeton and Youngs' experiment Case 110.

GRAV is the earth's nominal gravity at sea level, $980 \mathrm{e}-12\left(\mathrm{~cm}^{2} / \operatorname{microsec}^{2}\right)$. We use the Ares time-dependent, additive vertical acceleration source function, yasrc4, to provide the acceleration.

Time (microsec) Vertical accel. $\left(\mathrm{cm}^{2} /\right.$ microsec $\left.^{2}\right)$

$0.0 \mathrm{e} 3 \quad\{-0.000 * \mathrm{GRAV}\}$

$2.0 \mathrm{e} 3\{-0.000 * \mathrm{GRAV}\}$

$3.0 \mathrm{e} 3 \quad\left\{-1.018^{*} \mathrm{GRAV}\right\}$

$4.0 \mathrm{e} 3 \quad\left\{-3.258^{*} \mathrm{GRAV}\right\}$

$5.0 \mathrm{e} 3 \quad\left\{-6.515^{*} \mathrm{GRAV}\right\}$

$6.0 \mathrm{e} 3\{-10.180 * \mathrm{GRAV}\}$

$7.0 \mathrm{e} 3\{-14.150 *$ GRAV $\}$

8.0e3 $\{-18.019 *$ GRAV $\}$

9.0e3 $\{-21.582 *$ GRAV $\}$

$10.0 \mathrm{e} 3\left\{-24.636^{*} \mathrm{GRAV}\right\}$

$11.0 \mathrm{e} 3\left\{-26.875^{*} \mathrm{GRAV}\right\}$

$12.0 \mathrm{e} 3\left\{-28.809^{*} \mathrm{GRAV}\right\}$

$13.0 \mathrm{e} 3\left\{-30.540^{*} \mathrm{GRAV}\right\}$

$14.0 \mathrm{e} 3\left\{-32.067^{*} \mathrm{GRAV}\right\}$

$15.0 \mathrm{e} 3\left\{-33.187^{*} \mathrm{GRAV}\right\}$

$16.0 \mathrm{e} 3\{-34.103 * \mathrm{GRAV}\}$

$17.0 \mathrm{e} 3\left\{-34.816^{*} \mathrm{GRAV}\right\}$

$18.0 \mathrm{e} 3\left\{-35.426^{*} \mathrm{GRAV}\right\}$

$19.0 \mathrm{e} 3\left\{-35.732^{*} \mathrm{GRAV}\right\}$

$20.0 \mathrm{e} 3\left\{-36.037^{*} \mathrm{GRAV}\right\}$

$21.0 \mathrm{e} 3\left\{-36.343^{*} \mathrm{GRAV}\right\}$

$22.0 \mathrm{e} 3\left\{-36.444^{*} \mathrm{GRAV}\right\}$

$23.0 \mathrm{e} 3\left\{-36.546^{*} \mathrm{GRAV}\right\}$

$24.0 \mathrm{e} 3\{-36.750 * \mathrm{GRAV}\}$

$25.0 \mathrm{e} 3\left\{-36.648^{*} \mathrm{GRAV}\right\}$

$80.0 \mathrm{e} 3\{-34.510 * \mathrm{GRAV}\}$

81.0e3 $\left\{-34.307^{*} \mathrm{GRAV}\right\}$

$82.0 \mathrm{e} 3\left\{-34.103^{*} \mathrm{GRAV}\right\}$

83.0e3 $\{-33.289 *$ GRAV $\}$

84.0e3 $\{-32.169 * \mathrm{GRAV}\}$

85.0e3 $\left\{-30.744^{*} \mathrm{GRAV}\right\}$

1.e10 $\left\{-30.744^{*} \mathrm{GRAV}\right\}$ 